\title{
Neutrino scattering and $B$ anomalies from hidden sector portals
}

\author{
Alakabha Datta, ${ }^{a, b}$ Bhaskar Dutta, ${ }^{c}$ Shu Liao, ${ }^{c}$ Danny Marfatia ${ }^{d}$ and Louis E. Strigari ${ }^{c}$ \\ ${ }^{a}$ Department of Physics and Astronomy, University of Mississippi, \\ 108 Lewis Hall, Oxford, MS 38677-1848, U.S.A. \\ ${ }^{b}$ Department of Physics and Astronomy, University of California, \\ 4129 Frederick Reines Hall, Irvine, CA 92697-4575, U.S.A. \\ ${ }^{c}$ Mitchell Institute for Fundamental Physics and Astronomy, \\ Department of Physics and Astronomy, Texas A $6 M$ University, \\ 4242 TAMU, College Station, TX 77845, U.S.A. \\ ${ }^{d}$ Department of Physics and Astronomy, University of Hawaii-Manoa, \\ 2505 Correa Road, Honolulu, HI 96822, U.S.A. \\ E-mail: datta@phy.olemiss.edu, dutta@physics.tamu.edu, \\ ikaros@physics.tamu.edu, dmarf8@hawaii.edu, strigari@tamu.edu
}

ABSTRACT: We examine current constraints on and the future sensitivity to the strength of couplings between quarks and neutrinos in the presence of a form factor generated from loop effects of hidden sector particles that interact with quarks via new interactions. We consider models associated with either vector or scalar interactions of quarks and leptons generated by hidden sector dynamics. We study constraints on these models using data from coherent elastic neutrino-nucleus scattering and solar neutrino experiments and demonstrate how these new interactions may be discovered by utilizing the recoil spectra. We show that our framework can be naturally extended to explain the lepton universality violating neutral current $B$ decay anomalies, and that in a model framework the constraints from neutrino scattering can have implications for these anomalies.

Keywords: Beyond Standard Model, Neutrino Physics, Kaon Physics, Heavy Quark Physics

ArXiv ePrint: 1808.02611 


\section{Contents}

1 Introduction 1

2 Form factors 2

$2.1 Z^{\prime}$ model 2

$\begin{array}{ll}2.2 S \text { model } & 4\end{array}$

3 Scattering cross sections 5

4 Experimental bounds $\quad 6$

$\begin{array}{llr}4.1 & \text { Accelerators and reactors } & 6\end{array}$

$\begin{array}{llr}4.2 & \text { Solar neutrinos } & 8\end{array}$

$5 \quad$ B anomalies $\quad 9$

6 Conclusions $\quad 15$

\section{Introduction}

In spite of the success of the standard model (SM) in describing the particle interactions observed in nature, neutrino interactions with matter are not thoroughly understood. Many experiments are now making precise measurements of neutrino-nucleus scattering cross sections and neutrino-electron elastic scattering cross sections. The measurements are now precise enough that they are able to probe beyond the SM physics. Recently, the COHERENT experiment at the Spallation Neutron Source (SNS) has measured coherent elastic neutrino-nucleus scattering ( $\mathrm{CE} \nu \mathrm{NS})$ for the first time, finding that the cross section for scattering on CsI is consistent with the SM at approximately $1 \sigma$ [1]. In addition, measurements by Borexino of the solar neutrino flux, in particular the ${ }^{7} \mathrm{Be}$ component, now provide the best measurement of the neutrino-electron elastic scattering cross section at electron recoil energies $\lesssim \mathrm{MeV}[2]$.

Because of this plethora of current and future experimental data, it is imperative to consider new theoretical ideas for neutrino interactions in these low energy experiments. Consider axial-vector interactions between quarks and neutrinos mediated by a new $Z^{\prime}$ boson. We write the interaction of the quarks with the $Z^{\prime}$ as,

$$
\mathcal{L}_{q^{\prime} q^{\prime}}=\overline{q^{\prime}} \hat{\gamma}^{\mu}\left[P_{L} F_{L}\left(q^{2}\right)+P_{R} F_{R}\left(q^{2}\right)\right] q^{\prime} Z_{\mu}^{\prime},
$$

where $\hat{\gamma}^{\mu}=\left[\gamma_{\mu}-\frac{\gamma \cdot q q^{\mu}}{q^{2}}\right]$ and $q^{\prime}$ are SM quark fields. The interaction of the $Z^{\prime}$ with the leptons has an analogous expression. In the interaction above, the contribution from the $q^{\mu}$ part may be suppressed by small masses or vanish from current conservation. Form 
factors proportional to $\sigma^{\mu \nu} q_{\nu}$ are possible, but will be suppressed by some hadronic scale; we do not investigate these in this paper. The form factor $F\left(q^{2}\right)$ can be unity when $q^{\prime}$ couples directly to $Z^{\prime}$. However, in many models (especially the models with low scale hidden sectors), $q^{\prime}$ may couple to $Z^{\prime}$ via a loop containing DM/hidden sector particles. In such a scenario we expect $F\left(q^{2}\right) \sim q^{2} / \Lambda^{2}$ where $\Lambda$ is the scale in the DM sector associated with the mass of the mediator particle that generates the quark-DM interactions, $\bar{q} q \bar{\chi} \chi$. As long as $\Lambda$ is greater than $q_{\text {max }}$ for these scattering experiments, $F\left(q^{2}\right) \sim q^{2} / \Lambda^{2}$ appears in the scattering amplitude. In this paper, we investigate these new form factors at $\mathrm{CE} \nu \mathrm{NS}$ (COHERENT and reactor based) and Borexino experiments for vector and scalar mediators. We extend our framework to study the neutral current $B$ decay anomalies in the $R_{K}$ and $R_{K^{*}}$ measurements. We show how in a model framework measurements from neutrino scattering may have implications for the $B$ anomalies.

The paper is organized as follows. In section 2, we discuss models for form factors and their dependence on the new physics scale. In sections 3 and 4 we discuss the effects of these new form factors at $\mathrm{CE} \nu \mathrm{NS}$ and Borexino experiments, respectively. In section 5 we discuss the $B$ anomalies and the implications of neutrino scattering experiments on their explanations. We conclude in section 6 .

\section{Form factors}

\section{$2.1 \quad Z^{\prime}$ model}

Consider the following Lagrangian at low energy [3]:

$$
\begin{aligned}
\mathcal{L} & =\frac{g}{\Lambda^{2}} \overline{q^{\prime}} \gamma^{\mu} P_{L, R} q^{\prime} \bar{\chi} \gamma_{\mu}\left(1 \pm \gamma_{5}\right) \chi+i \bar{\chi} \gamma^{\nu}\left[\partial_{\nu}-i g_{\chi} Z^{\prime \nu}\right] \chi-m_{\chi} \bar{\chi} \chi+\frac{1}{2} m_{Z^{\prime}}^{2} Z_{\mu}^{\prime} Z^{\prime \mu} \\
& =H_{\mathrm{eff}}+J_{\mu, \chi} Z^{\prime, \mu}+i \bar{\chi} \gamma^{\nu} \partial_{\nu} \chi-m_{\chi} \bar{\chi} \chi+\frac{1}{2} m_{Z^{\prime}}^{2} Z_{\mu}^{\prime} Z^{\prime \mu}
\end{aligned}
$$

where $\chi$ is a hidden sector fermion field with mass $m_{\chi}$. The first term in the Lagrangian represents an effective coupling between the $q$ and $\chi$ fields that might arise through the exchange of a heavy mediator of mass $\sim \Lambda$, with $\Lambda \gg E$, where $E$ is the energy scale of the process (see for example [4-6]). The hidden sector fields $\chi$ couple directly to $Z^{\prime}$ through the vector portal and so in our framework there are two mediators; see e.g., [7]. We further assume that the neutrinos are charged under the $Z^{\prime} \mathrm{U}(1)$ and so there is a direct coupling of the neutrinos to $Z^{\prime}$. Although there is no direct coupling between the quarks and $Z^{\prime}$ field, a $\chi$-loop-induced $\bar{q} q Z^{\prime}$ effective vertex (as in eq. 1.1) will be generated with a $q^{2}$ dependent coupling, which can be represented by a higher dimensional operator,

$$
\mathcal{L}_{H D}=\frac{g_{L, R}}{\Lambda^{2}} \bar{q}^{\prime} \gamma^{\mu} P_{L, R} q^{\prime} \partial^{\nu} Z_{\mu \nu}^{\prime},
$$

where $Z_{\mu \nu}^{\prime}$ is the $Z^{\prime}$ field tensor; see figure 1 . This higher dimensional operator may be considered to be the bare term of the Lagrangian.

The form factors in eq. (1.1) are then given by

$$
\overline{q^{\prime}} \hat{\gamma^{\mu}}\left[P_{L} F_{L}\left(q^{2}\right)+P_{R} F_{R}\left(q^{2}\right)\right] q^{\prime}=\frac{1}{\Lambda^{2}}\left\langle q^{\prime}\left|\int d^{4} x e^{i q \cdot x} T\left[J_{\mu, \chi}(x) H_{\mathrm{eff}}(0)\right]\right| q^{\prime}\right\rangle .
$$




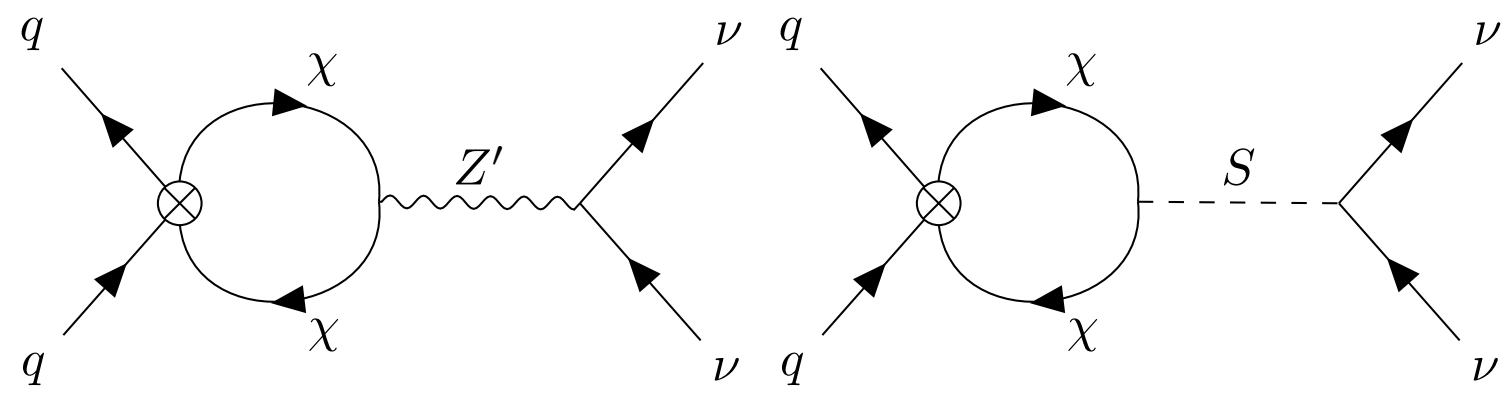

Figure 1. Feynman diagram for $Z^{\prime}$ model (left) and $S$ model (right).

The most general structure for the form factors from the current conservation of $J_{\mu, \chi}$ is

$$
\begin{aligned}
& F_{L}\left(q^{2}\right)=\frac{q^{2}}{\Lambda^{2}} A_{L}\left(q^{2}\right), \\
& F_{R}\left(q^{2}\right)=\frac{q^{2}}{\Lambda^{2}} A_{R}\left(q^{2}\right) .
\end{aligned}
$$

Since the matrix element in eq. (2.3) is finite as $q^{2} \rightarrow 0, A_{L, R}\left(q^{2}\right)$ is finite as $q^{2} \rightarrow 0$. Now from eqs. (2.2) and (2.3), we can estimate the form factor in the 1-loop approximation [8]. We get

$$
F_{L, R}\left(q^{2}\right)=\frac{q^{2}}{\Lambda^{2}}\left[g_{L, R}+g g_{\chi} \frac{1}{(2 \pi)^{4}} \int_{0}^{1} d x 8 x(1-x) \int d^{4} l \frac{1}{\left(l^{2}-\Delta+i \epsilon\right)^{2}}\right],
$$

where $\Delta=m_{\chi}^{2}-q^{2} x(1-x)$. Introducing a cut-off $\Lambda_{c}$ to regulate the divergent integral we can write

$$
\begin{aligned}
F_{L, R}\left(q^{2}\right) & =\frac{q^{2}}{\Lambda^{2}}\left[g_{L, R}+\frac{g g_{\chi}}{2 \pi^{2}} \int_{0}^{1} d x x(1-x) \ln \frac{x \Lambda_{c}^{2}}{\Delta}\right], \\
& =\frac{q^{2}}{\Lambda^{2}} g_{L, R}\left(q^{2}\right) .
\end{aligned}
$$

where $g$ and $g_{\chi}$ are the bare coupling constants. We rewrite $g_{L, R}\left(q^{2}\right)$ as

$$
g_{L, R}\left(q^{2}\right)=g_{L, R}\left(q_{\max }^{2}\right)+\frac{g g_{\chi}}{2 \pi^{2}} \int_{0}^{1} d x x(1-x) \ln \frac{\Delta_{\max }}{\Delta},
$$

where $\Delta_{\max }=m_{\chi}^{2}-q_{\max }^{2} x(1-x)$ and $q_{\max }^{2}$ is the maximum momentum transfer squared.

Note that this is a rough estimate as we have calculated only the leading term in eq. (2.3). However, the general structure of eq. (2.4) still holds as it follows from vector current conservation. As a rough estimate, assuming all terms in eq. (2.7) to be of the same size, we can write,

$$
g_{L, R}\left(q^{2}\right) \sim \frac{g g_{\chi}}{2 \pi^{2}} \int_{0}^{1} d x x(1-x) \ln \frac{\Delta_{\max }}{\Delta} .
$$

For our purposes, the most important part of eq. (2.5) is the $q^{2} / \Lambda^{2}$ factor which is not present if either the tree level (first term) or the loop contribution (second term) is 
absent; the latter is eq. (2.8). We show both tree and loop contributions since each term may not be sizable in a given model. The relative strength between the tree and the loop contributions does not affect our analysis.

Although the Lagrangian in eq. (2.1) contains both $g_{L}$ and $g_{R}$ terms, only $g_{L}+g_{R} \equiv g_{v}$ contributes to $\nu$-nucleus coherent scattering; $g_{L}-g_{R} \equiv g_{a}$ does not impact the scattering process. This is because the vector charge of the nucleus is proportional to the number of nucleons, $Q_{v}=Z g_{v}^{p}+N g_{v}^{n}$ while the axial vector couplings are proportional to the spin, $Q_{a}=g_{a}^{p}\left\langle S_{p}\right\rangle+g_{a}^{n}\left\langle S_{n}\right\rangle$ which is much smaller than $Q_{v}$.

\section{$2.2 S$ model}

As for the $Z^{\prime}$ case the form factors for a scalar mediator $S$ can be written as

$$
\overline{q^{\prime}}\left[P_{L} S_{L}\left(q^{2}\right)+P_{R} S_{R}\left(q^{2}\right)\right] q^{\prime}==\frac{1}{\Lambda^{2}}\left\langle q^{\prime}\left|\int d^{4} x e^{i q \cdot x} T\left[J_{\chi}(x) H_{\mathrm{eff}}(0)\right]\right| q^{\prime}\right\rangle,
$$

where

$$
\begin{aligned}
H_{\mathrm{eff}} & =\frac{g}{\Lambda^{2}} \bar{q} P_{L, R} q \bar{\chi}\left(1 \pm \gamma_{5}\right) \chi, \\
J_{\chi} & =\bar{\chi}\left[g_{\chi} P_{L}+g_{\chi}^{\prime} P_{R}\right] \chi .
\end{aligned}
$$

In this case we cannot use current conservation. By calculating the leading loop contribution we find,

$$
S_{L, R}\left(q^{2}\right)=g g_{\chi} \frac{1}{(2 \pi)^{4}} \int_{0}^{1} d x 12 x(1-x) \frac{\Delta}{\Lambda^{2}} \int d^{4} l \frac{1}{\left(l^{2}-\Delta+i \epsilon\right)^{2}} .
$$

Again introducing a cut-off $\Lambda_{c}$ we can write,

$$
S_{L, R}\left(q^{2}\right) \sim \frac{3 g g_{\chi}}{4 \pi^{2}} \int_{0}^{1} d x x(1-x) \frac{\left[m_{\chi}^{2}-q^{2} x(1-x)\right]}{\Lambda^{2}} \ln \frac{x \Lambda_{c}^{2}}{\Delta} .
$$

For $m_{\chi}^{2} \ll q^{2}, S_{L, R}\left(q^{2}\right) \sim q^{2}$. Unlike the $Z^{\prime}$ case the form factor is sensitive to the $\chi$ mass and so the hidden sector dynamics can be probed in low energy scattering.

A scalar coupling has a larger effect on the coherent scattering rate $\sim O\left(E_{R}\right)$, compared to the rate from a pseudoscalar coupling, $\frac{1}{8 \pi p^{2}} \frac{g_{\nu}^{2} g_{q}^{2}}{\left(2 m_{N} E_{R}+m_{s}^{2}\right)^{2}} E_{R}^{2} m_{N} \sim O\left(E_{R}^{2}\right)$.

The scalar interactions of quarks with dark matter $\sim \frac{g}{\Lambda^{2}} \bar{q} P_{L, R} q \bar{\chi} P_{L, R} \chi$ could arise from a higher dimensional operator $\frac{g}{\Lambda^{3}} H \bar{q} P_{L, R} q \bar{\chi} P_{L, R} \chi$. With the assumption of minimal flavor violation (MFV) this interaction has the form $\sim \frac{m_{q}}{\Lambda^{3}} \bar{q} P_{L, R} q \bar{\chi} P_{L, R} \chi[4]$.

It is worth considering how the phenomenology changes if one uses MFV to constrain the scalar interactions. Without MFV the matrix element that appears in the amplitude is $M \sim\langle N|\bar{q} q| N\rangle$ while with MFV we need $M^{\prime} \sim \frac{1}{\Lambda}\left\langle N\left|m_{q} \bar{q} q\right| N\right\rangle$. If the heavy $c, b, t$ quarks are involved they can be integrated out and their contributions replaced by a gluonic term [9, 10]. If contributions arise only from the light quarks then $\frac{M^{\prime}}{M} \sim \frac{m_{q}}{\Lambda}$, and for $\Lambda \gg m_{q}$ the matrix element for the MFV case is suppressed. Hence, the bounds on $\Lambda$ from coherent scattering will be weaker.

When we discuss the $B$ anomalies below, we will require that dark matter couple to quarks of all generations or at least to all generations of down quarks. Consequently, the 
heavy quarks could contribute to coherent scattering via the gluonic terms. However, for the $B$ anomalies we need a flavor changing coupling $g_{b s}$ which involves a mixing angle. In other words, the $B$ anomalies do not completely fix the coupling $g_{q}$, of the heavy quarks to dark matter. The heavy quark contribution to the matrix element $M$ goes as $\sim g_{q} \frac{m_{N}}{m_{q}}$ while for $M^{\prime}$ it goes as $\sim g_{q} \frac{m_{N}}{\Lambda}$, where $m_{N}$ is the nucleon mass.

Note that the scalar coupling to the neutrinos may originate from the lepton number violating interaction, $\phi \bar{\nu}_{L}^{c} \nu_{L}$, or from the lepton number conserving interaction, $\phi \bar{\nu}_{R} \nu_{L}$, where $\phi$ is a SM scalar singlet. If $\phi$ is a pseudoscalar, then the associated coupling is not constrained by coherent scattering but is constrained by $\pi^{0} \rightarrow \bar{\nu} \nu$ [11] to be smaller than $10^{-5}$.

Finally, depending on whether or not right-handed neutrinos are present, we can write neutrino-quark interaction terms as $g^{\prime} / m^{\prime 2} \bar{\nu}_{R} \nu_{L} \bar{q} q$ or $g^{\prime} / m^{\prime 2} \bar{\nu}_{L}^{c} \nu_{L} \bar{q} q$. If the second term is present, then a Majorana mass term arises from the quark condensate, i.e., $m_{\nu}=g^{\prime} /\left(m^{\prime} / \mathrm{GeV}\right)^{2} \bar{q} q=g^{\prime} /\left(m^{\prime} / \mathrm{GeV}\right)^{2} 10^{7} \mathrm{eV}$ with $\bar{q} q=8 \pi / \sqrt{3} f_{\pi}^{3}$ as calculated in the Nambu-Jona-Lasinio model [12]. Since the heaviest neutrino mass is $\sim 0.1 \mathrm{eV}$, we find $g^{\prime} /\left(m^{\prime} / \mathrm{GeV}\right)^{2} \leq 10^{-8}$. However, $g^{\prime}$ is constrained by COHERENT to be smaller than $10^{-10}\left(10^{-8}\right)$ for $m^{\prime}=10 \mathrm{MeV}(1 \mathrm{GeV})[13]$.

\section{Scattering cross sections}

For the vector model we can write the effective interaction as

$$
\mathcal{L}_{\mathrm{BSM}}=-2 \sqrt{2} G_{F} \overline{\nu_{L}} \gamma^{\mu} \nu_{L} \bar{f} \gamma_{\mu} f \frac{g \prime F\left(q^{2}, \Lambda^{2}\right)}{q^{2}+m^{\prime 2}} \frac{1}{2 \sqrt{2} G_{F}},
$$

where $F\left(q^{2}, \Lambda^{2}\right)=\frac{q^{2}}{\Lambda^{2}}$. Since the vector interaction has the same structure as in the SM, its contribution can interfere with the SM contribution.

The neutrino-electron differential cross section can be written as

$$
\frac{d \sigma}{d E_{R}}=\frac{2}{\pi} G_{F}^{2} m_{e}\left[\left(\epsilon^{L}\right)^{2}+\left(\epsilon^{R}\right)^{2}\left(1-\frac{E_{R}}{E_{\nu}}\right)^{2}-\epsilon^{L} \epsilon^{R} \frac{m_{e} E_{R}}{E_{\nu}^{2}}\right],
$$

where $\epsilon^{L}=\frac{1}{2}+\sin ^{2} \theta_{w}+\frac{g^{\prime} F\left(q^{2}, \Lambda^{2}\right)}{q^{2}+m^{\prime 2}} \frac{1}{2 \sqrt{2} G_{F}}$ and $\epsilon^{R}=\sin ^{2} \theta_{w}+\frac{g \prime F\left(q^{2}, \Lambda^{2}\right)}{q^{2}+m^{\prime 2}} \frac{1}{2 \sqrt{2} G_{F}}$ for the electron neutrino, and $\epsilon^{L}=-\frac{1}{2}+\sin ^{2} \theta_{w}+\frac{g^{\prime} F\left(q^{2}, \Lambda^{2}\right)}{q^{2}+m^{\prime 2}} \frac{1}{2 \sqrt{2} G_{F}}$ and $\epsilon^{R}=\sin ^{2} \theta_{w}+\frac{g \prime F\left(q^{2}, \Lambda^{2}\right)}{q^{2}+m^{\prime 2}} \frac{1}{2 \sqrt{2} G_{F}}$ for the muon or tau neutrino.

The neutrino-nucleus differential cross section is

$$
\frac{d \sigma}{d E_{R}}=\frac{2}{\pi} G_{F}^{2} m_{N} Q_{V}^{2}\left[1-\frac{m_{N} E_{R}}{E_{\nu}^{2}}+\left(1-\frac{E_{R}}{E_{\nu}}\right)^{2}\right] F_{\text {nucl }}\left(q^{2}\right),
$$

where $F_{\text {nucl }}\left(q^{2}\right)$ is the nuclear form factor, the "weak charge" $Q_{V}$ is given by

$$
Q_{V}=\frac{1}{2}\left[Z\left(\frac{1}{2}-2 \sin ^{2} \theta_{w}\right)+N\left(-\frac{1}{2}\right)+3(Z+N) \frac{g \prime F\left(q^{2}, \Lambda^{2}\right)}{q^{2}+m^{\prime 2}} \frac{1}{2 \sqrt{2} G_{F}}\right],
$$

We have assumed the $Z^{\prime}$ couplings are the same for the up and down quark. 
On the other hand, for the scalar model, the effective interaction is

$$
\mathcal{L}_{\mathrm{BSM}}=\bar{\nu}_{L}^{c} \nu_{L} \bar{f} f \frac{g \prime F\left(q^{2}, \Lambda^{2}\right)}{q^{2}+m^{\prime 2}},
$$

which does not interfere with the SM. We can write its contribution to the differential cross section as

$$
\frac{d \sigma}{d E_{R}}=\left.\frac{d \sigma}{d E_{R}}\right|_{\mathrm{SM}}+\frac{1}{4 \pi}\left(\frac{g \prime F\left(q^{2}, \Lambda^{2}\right)}{q^{2}+m^{\prime 2}}\right)^{2} \frac{E_{R} m_{e}^{2}}{E_{\nu}^{2}},
$$

for electron scattering, and

$$
\frac{d \sigma}{d E_{R}}=\left.\frac{d \sigma}{d E_{R}}\right|_{\mathrm{SM}}+\frac{1}{4 \pi}\left(\frac{(15.1 Z+14 N) g \prime F\left(q^{2}, \Lambda^{2}\right)}{q^{2}+m^{\prime 2}}\right)^{2} \frac{E_{R} m_{e}^{2}}{E_{\nu}^{2}},
$$

for nucleus scattering [9]. Here $Z(N)$ corresponds to the number of protons (neutrons).

\section{Experimental bounds}

This section describes the experiments that we use to bound the aforementioned models. We begin by discussing accelerator and reactor experiments, and then discuss solar neutrino experiments.

\subsection{Accelerators and reactors}

To evaluate current and future constraints from accelerator and reactor $\mathrm{CE} \nu \mathrm{NS}$ experiments, we use a $\chi^{2}$ analysis to calculate bounds on the coupling at the $2 \sigma$ confidence level. To take into account reactor and accelerator neutrino flux uncertainties, we introduce a nuisance parameter $\alpha$ and an uncertainty on the signal of $\sigma_{\alpha}$. We define

$$
\chi^{2}=\sum_{\text {bins }}\left[\frac{N_{\mathrm{obs}}^{i}-(1+\alpha) N_{\mathrm{th}}^{i}}{\sigma_{\text {stat }}^{i}}\right]^{2}+\left(\frac{\alpha}{\sigma_{\alpha}}\right)^{2},
$$

where $N_{\text {obs }}^{i}\left(N_{\text {th }}^{i}\right)$ is the observed (predicted) number of events per bin in a current measurement, $\sigma_{\alpha}=0.28$ and $\sigma_{\text {stat }}^{i}$ is the statistical uncertainty which can be extracted from ref. [1]. For future measurements with multiple detectors we define (with indices suppressed),

$$
\chi^{2}=\sum_{\text {bins, detectors }} \frac{\left(N_{\mathrm{SM}}-(1+\alpha) N_{\mathrm{th}}\right)^{2}}{N_{b g}+N_{\mathrm{SM}}}+\left(\frac{\alpha}{\sigma_{\alpha}}\right)^{2},
$$

where $N_{\mathrm{SM}}$ is the expected number of events in the SM for a future measurement and $N_{b g}$ is the expected number of background events, which we assume to be known precisely. Here we estimate $\sigma_{\alpha}=0.1$ for future measurement.

The current COHERENT experiment has a threshold $4.25 \mathrm{keV}$ [1]. For the future projected measurements we assume a threshold of $100 \mathrm{eV}$ for Ge and Si reactor experiments [14-16], and $2 \mathrm{keV}$ for $\mathrm{NaI}$ and $\mathrm{Ar}$ with COHERENT [17]. For reactor neutrinos we take a background of $1 \mathrm{dru}(\mathrm{Ge}$ and $\mathrm{Si}$ ), and for accelerator neutrino data we take a 


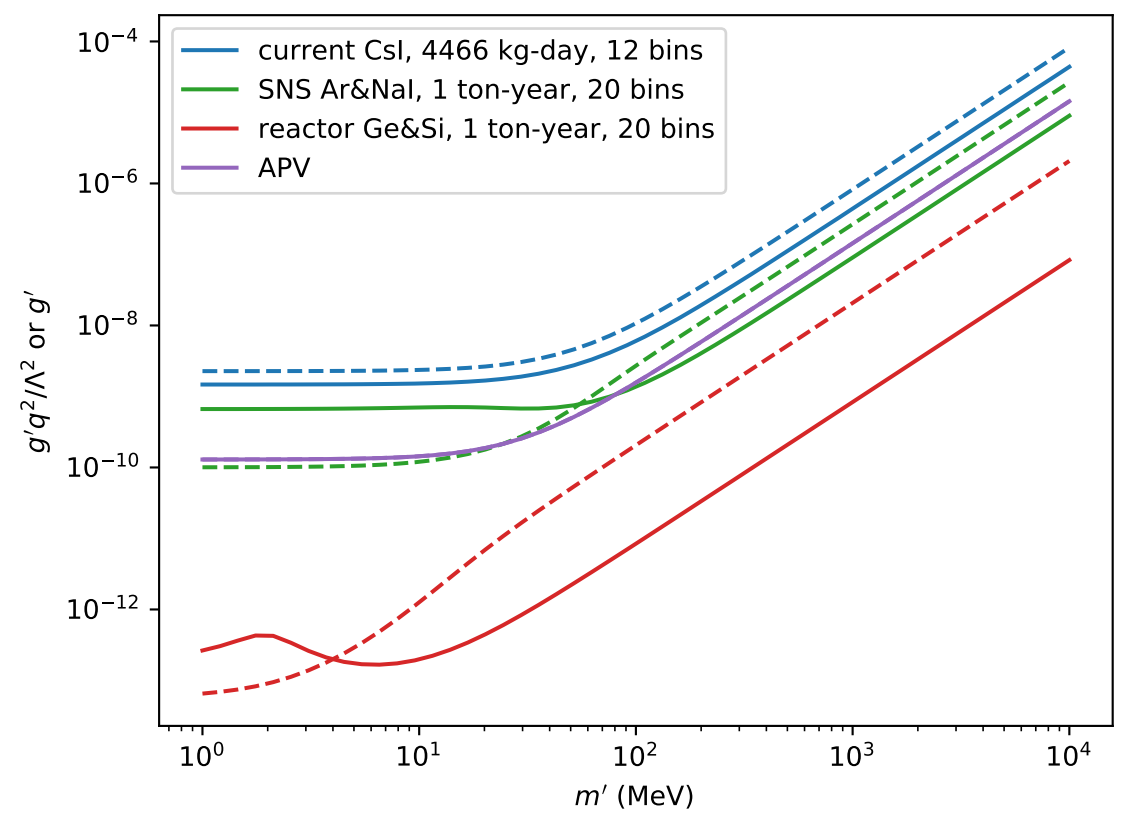

Figure 2. Current and projected $2 \sigma$ bounds on a vector mediator with $F\left(q^{2}\right) \sim q^{2}$ as a function of the mediator mass. Dashed lines show the limits without a form factor. Here $q_{0}=50 \mathrm{MeV}$ for COHERENT, and $q_{0}=30 \mathrm{MeV}$ for reactor experiments.

background of $5 \times 10^{-3} \mathrm{dru}$ (CsI, NaI and Ar) [1]. Here the unit dru stands for differential rate unit, equal to event/ $(\mathrm{keV} \cdot \mathrm{kg} \cdot$ day $)$. The COHERENT experiment has an energy dependent efficiency. We applied the efficiency function from [1] to all the detectors in the COHERENT experiment. We take the reactor neutrino flux to be that of a $1 \mathrm{MW}$ reactor at $\sim 1 \mathrm{~m}$ from the core (which yields a the total flux of $1.5 \times 10^{12} \mathrm{~cm}^{2} / \mathrm{s}$ ), and the antineutrino fission spectrum at various sites from ref. [18]. The accelerator neutrino flux at SNS is $4.29 \times 10^{9} \mathrm{~cm}^{2} / \mathrm{s}[1]$.

In figures 2 and 3 we show the COHERENT and reactor constraints on $\frac{g^{\prime} q^{2}}{\Lambda^{2}}=$ $\frac{\left(\left(g_{L}+g_{R}\right) g_{\nu}\right) q_{0}^{2}}{2 \Lambda^{2}}$ at $2 \sigma$ for a vector or scalar mediator, respectively, as a function of the mediator mass. $\frac{g^{\prime} q^{2}}{\Lambda^{2}}$ represents the coupling strength between quarks and neutrinos as a function of energy and reduces to $g^{\prime}$ if there is no form factor for the coupling. We choose $q_{0}$ to be a typical momentum for the experiment, e.g., $q_{0}=50 \mathrm{MeV}$ and $30 \mathrm{MeV}$ are used for COHERENT and reactor experiments, respectively. To compare with the limits for the case without a form factor, we plot the corresponding limits using dashed lines. The quarks may have direct couplings to the $Z^{\prime}$ and may also couple via DM loops in a given model, in which case the solid and dashed lines must be combined to obtain constraints on the couplings. The plateau for small mediator masses arises because $m^{\prime 2} \ll q^{2}$ which makes the limits independent of the mediator mass. In the regime of large mediator masses, the slope of the limit curves is 2 since the effective couplings become $\frac{g^{\prime}}{m^{\prime 2}}$, i.e., $\log g^{\prime} \propto 2 \log m^{\prime}$. Also notice that there is a bump in the low mass region for future COHERENT and reactor 


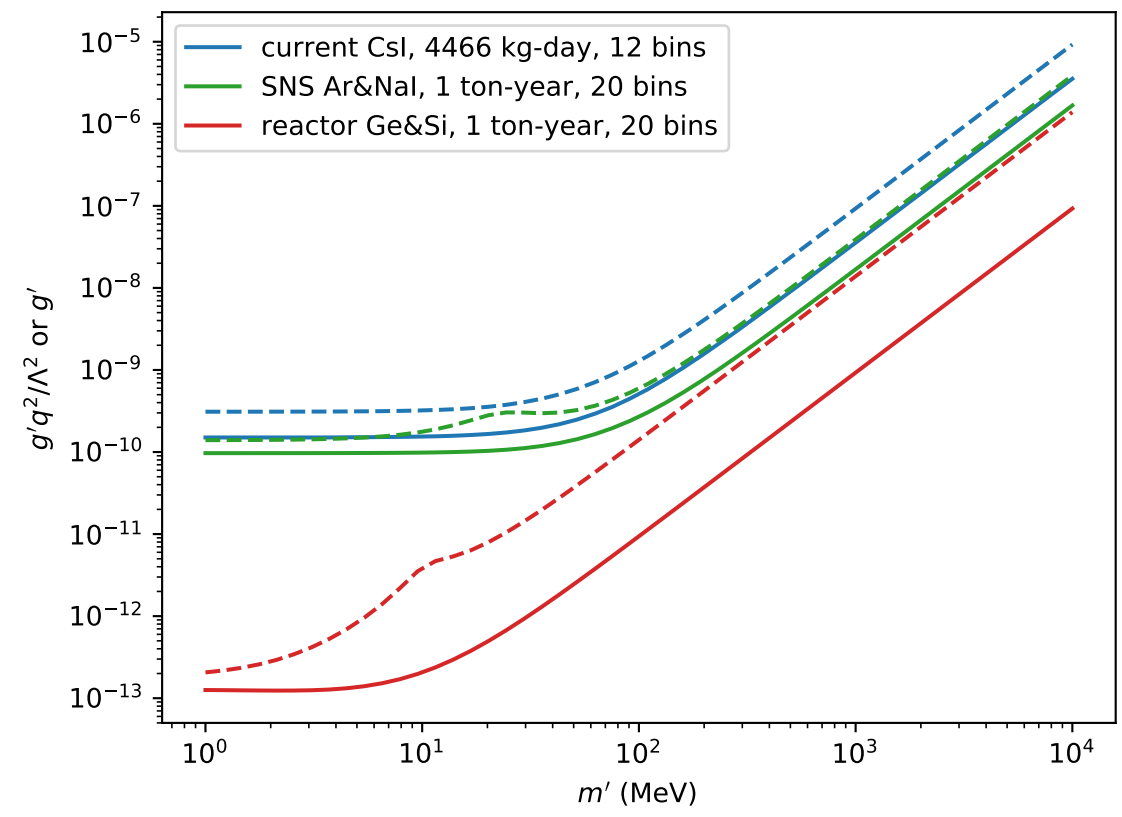

Figure 3. Current and projected $2 \sigma$ bounds on a scalar mediator with $F\left(q^{2}\right) \sim q^{2}$ as a function of the mediator mass. Dashed lines show the limits without a form factor. Here $q_{0}=50 \mathrm{MeV}$ for COHERENT, and $q_{0}=30 \mathrm{MeV}$ for reactor experiments.

experiments because a combination of the form factor and the mediator propagator yields $\frac{q^{2}}{q^{2}+m^{\prime 2}} \sim 1$, so that the mediator-induced spectral distortion is suppressed. On the other hand, for the case with no form factor, the shape distortion persists for low masses, which makes the limits stronger compared to the $F\left(q^{2}\right) \sim q^{2}$ case. Note that direct detection constraints are nonexistent for sub-GeV DM and collider bounds are nonexistent for a $\mathrm{GeV}$ mediator which allows a lot of the parameter space to be unconstrained for $g \leq 1$.

An effect of the form factor, $F\left(q^{2}\right) \sim q^{2}$, is that the spectral shapes differ from the SM prediction and from new physics models with $F\left(q^{2}\right)=1$. To illustrate this, we show the spectrum of coherent scattering off a Ar target in figure 4. We choose the coupling $g$ from current COHERENT constraints for $F\left(q^{2}\right) \sim q^{2}$ (solid line) and $F\left(q^{2}\right)=1$ (dashed line). The main difference between the solid lines and dashed lines are at the higher energy end because the form factor $q^{2}$ enhances the deviation from the SM. At low energy, the spectrum is suppressed by the detection efficiency.

\subsection{Solar neutrinos}

Several solar neutrino experiments, for example Super-K [19], SNO [20], and Borexino [2], are sensitive to the neutrino-electron elastic scattering detection channel. Since the typical momentum transfer that solar neutrino experiments are sensitive to is $\sim 0.4 \mathrm{MeV}$, it is possible to probe much smaller values of $\Lambda$ as compared to reactor and accelerator $\mathrm{CE} \nu \mathrm{NS}$ experiments. Here we consider all the most prominent low energy components of the solar 

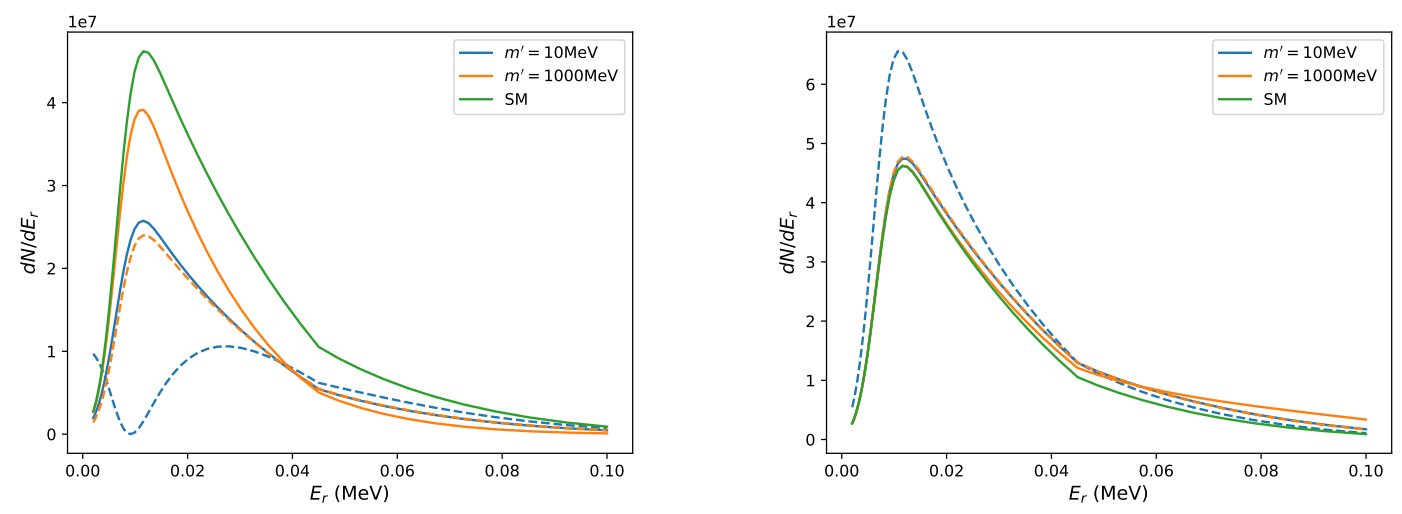

Figure 4. Spectrum of neutrino scattering off Ar detector with 1 ton-year exposure, with $\Lambda=$ $100 \mathrm{MeV}$. The left panel is for the vector mediator and the right panel is for the scalar mediator. Here the couplings for non-standard interactions are taken from the bound of current COHERENT CsI limit. The dashed lines show the spectrum without a form factor.

neutrino flux that Borexino is sensitive to, i.e., $p p$, pep, and ${ }^{7}$ Be. We choose the high metallicity solar model as defined in ref. [21] for our baseline Standard Solar Model (SSM), and comment on the impact of the model uncertainties below.

For solar neutrino experiments, the systematic uncertainties dominate. So we define $\chi^{2}$ for each component of the solar flux to be

$$
\chi^{2}=\frac{\left(N_{\mathrm{th}}-N_{\mathrm{obs}}\right)^{2}}{N_{\mathrm{obs}} \sigma},
$$

where $\sigma$ is the percent uncertainty in the measurement (including experimental and theoretical uncertainties in quadrature) with $\sigma_{p p}=0.11, \sigma_{7^{7} B e}=0.03$, and $\sigma_{p e p}=0.21$ [2]. To obtain a combined limit we define $\chi^{2}=\chi_{p p}^{2}+\chi_{7_{B e}}^{2}+\chi_{p e p}^{2}$.

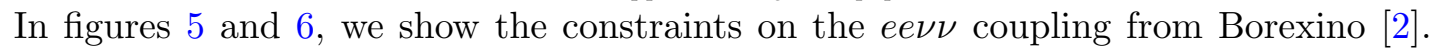
We find that the $p p$ and ${ }^{7} B e$ components provide the strongest constraints on $F\left(q^{2}\right) \sim q^{2}$ because of their higher event rates and smaller flux uncertainties. This is despite the fact that the pep component has larger spectral distortions (for the form-factor case relative to the $F\left(q^{2}\right)=1$ case) due to its higher energy. The limit plots are valid as long as $\Lambda^{2} \gg q^{2}$.

As for the nucleus scattering case, the recoil spectra in figure 7 show that the $F\left(q^{2}\right) \sim q^{2}$ case is different from the $F\left(q^{2}\right)=1$ case. We see that the major differences in the spectra are at high energies. The differences for the scalar case are more significant than for the vector case because in the vector scenario the $q^{2}$ enhancement is suppressed by the interference between SM and new physics contributions.

\section{B anomalies}

In the SM the three families of quarks and leptons are identical except for their masses. Tests of the universality of leptonic interactions are crucial probes of new physics. Recently, hints of lepton universality violating (LUV) measurements in $B$ decays have attracted a 


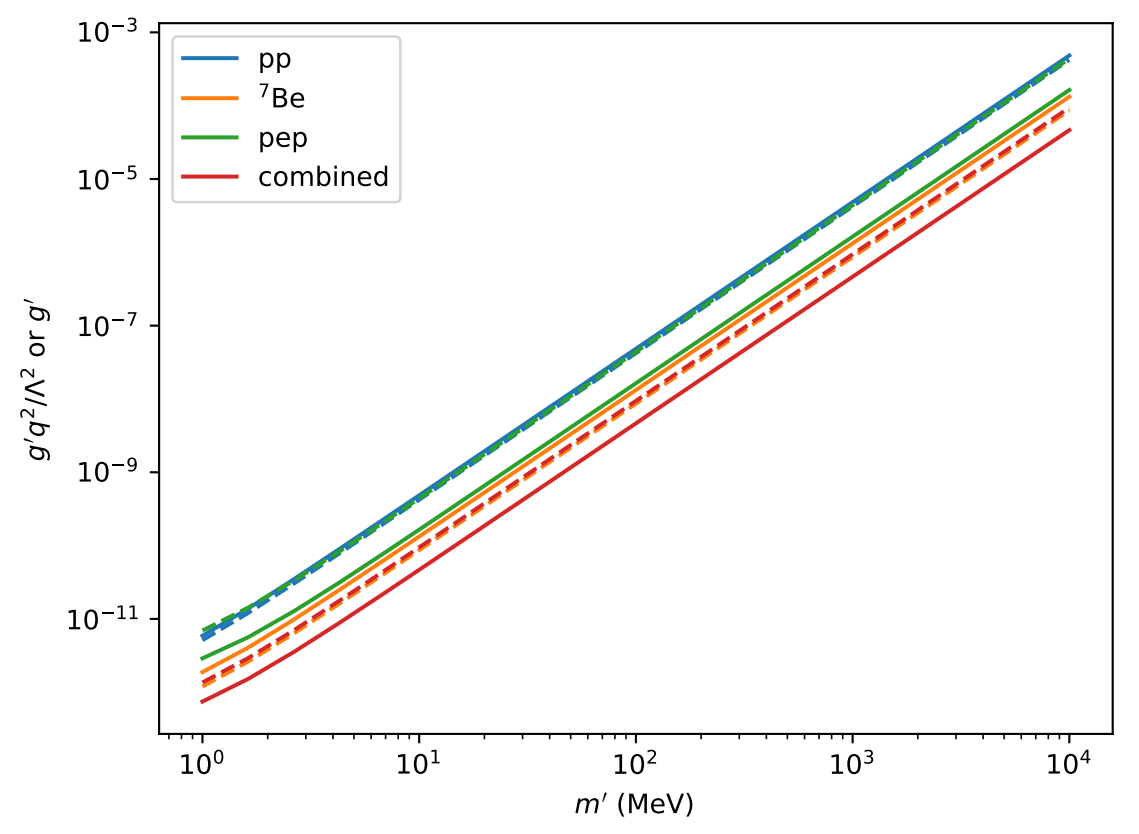

Figure 5. Constraints at $2 \sigma$ from the Borexino experiment on a vector mediator with $F\left(q^{2}\right) \sim q^{2}$ as a function of the mediator mass, compared to the case of a mediator without a form factor (dashed line). We set $q=0.5 \mathrm{MeV}$ and $\Lambda=10 \mathrm{MeV}$ for the form factor case to compare it to the no-form-factor case.

lot of attention. These anomalies are found in the charged current $b \rightarrow c \tau^{-} \bar{\nu}_{\tau}$ and neutral current $b \rightarrow s \ell^{+} \ell^{-}$transitions. Here we focus on the neutral current anomalies though the anomalies might be related [22-24]. The LHCb Collaboration has measured the ratio $R_{K^{*}} \equiv \mathcal{B}\left(B^{0} \rightarrow K^{* 0} \mu^{+} \mu^{-}\right) / \mathcal{B}\left(B^{0} \rightarrow K^{* 0} e^{+} e^{-}\right)$in two ranges of the dilepton invariant mass-squared $q^{2}[25]$ :

$$
R_{K^{*}}^{\text {expt }}=\left\{\begin{array}{c}
0.660_{-0.07}^{+0.11} \text { (stat) } \pm 0.03 \text { (syst), } \quad 0.045 \leq q^{2} \leq 1.1 \mathrm{GeV}^{2}, \quad\left(\text { low } q^{2}\right) \\
0.69_{-0.07}^{+0.11} \text { (stat) } \pm 0.05 \text { (syst), } \quad 1.1 \leq q^{2} \leq 6.0 \mathrm{GeV}^{2}, \quad\left(\text { central q } q^{2}\right)
\end{array}\right.
$$

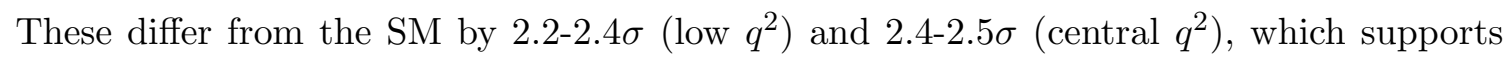
the hint of lepton nonuniversality observed earlier in a similar ratio with $K$ mesons. The observable in this case is $R_{K} \equiv \mathcal{B}\left(B^{+} \rightarrow K^{+} \mu^{+} \mu^{-}\right) / \mathcal{B}\left(B^{+} \rightarrow K^{+} e^{+} e^{-}\right)$[26, 27], which was measured by $\mathrm{LHCb}[28]$ :

$$
R_{K}^{\text {expt }}=0.745_{-0.074}^{+0.090} \text { (stat) } \pm 0.036 \text { (syst) }, \quad 1 \leq q^{2} \leq 6.0 \mathrm{GeV}^{2},
$$

and found to differ from the SM prediction, $R_{K}^{\mathrm{SM}}=1 \pm 0.01$ [29] by $2.6 \sigma$. Other anomalies also appear in the branching ratios and angular observables of certain $b \rightarrow s \mu^{+} \mu^{-}$decays. While many new physics models with new heavy states have been discussed to address these anomalies, it was pointed out that new physics with light mediators could also explain these anomalies [30]. In particular, with heavy new physics it is difficult to understand the $R_{K^{*}}$ 


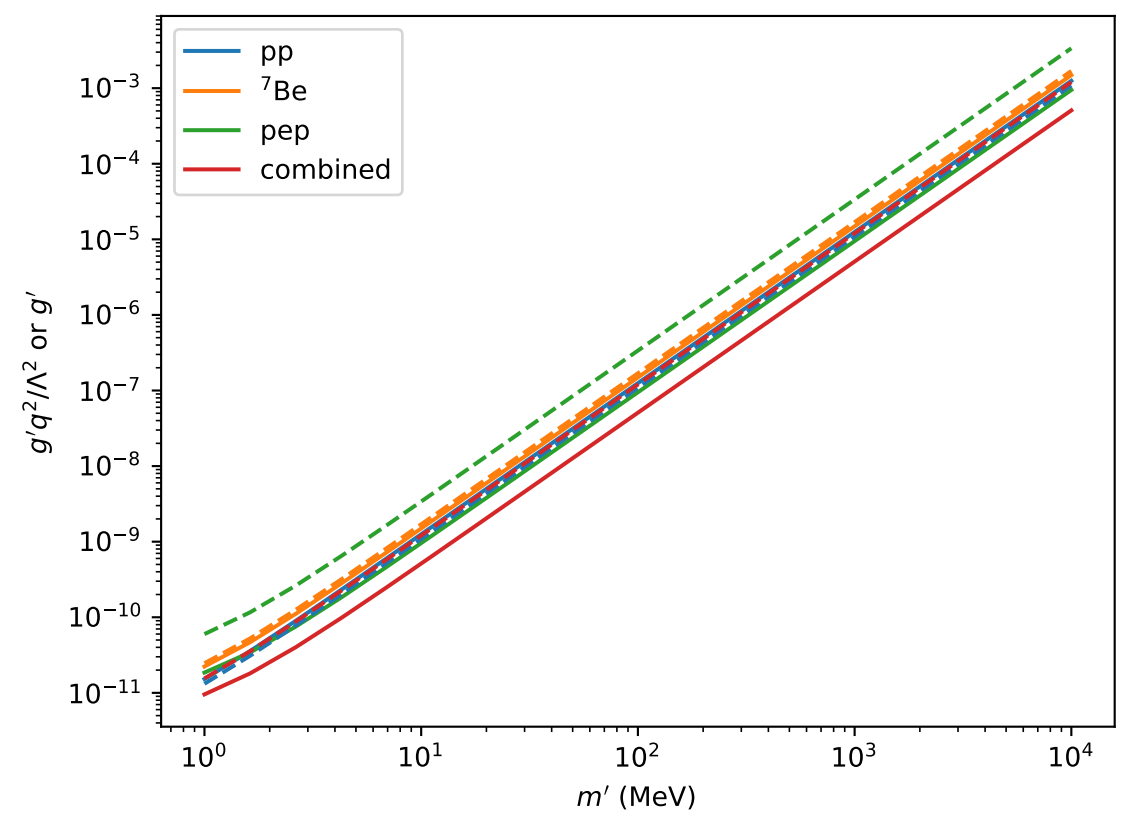

Figure 6. Constraints at $2 \sigma$ from the Borexino experiment on a scalar mediator with $F\left(q^{2}\right) \sim q^{2}$ as a function of the mediator mass, compared to the case of a mediator without a form factor (dashed line). We set $q=0.5 \mathrm{MeV}$ and $\Lambda=10 \mathrm{MeV}$ for the form factor case to compare it to the no-form-factor case.
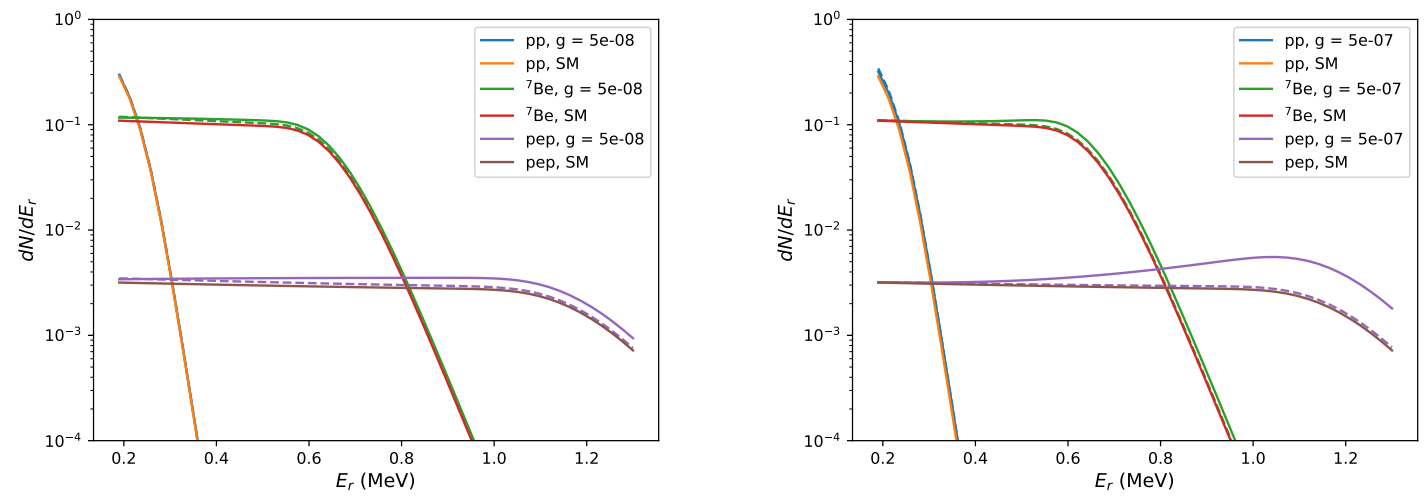

Figure 7. Spectra of solar neutrino scattering off electrons, with $m^{\prime}=10 \mathrm{MeV}$ and $\Lambda=10 \mathrm{MeV}$, and scaled to match the Borexino measurement. The left panel is for the vector mediator and the right panel is for the scalar mediator. Dashed lines are the spectra without a form factor. To make a fair comparison, for the latter case we scale $g$ by a factor of $q^{2} / \Lambda^{2}$. 
measurement in the low $q^{2}$ bin, $0.045 \leq q^{2} \leq 1.1 \mathrm{GeV}^{2}$, along with the measurement of $R_{K^{*}}$ in the central $q^{2}$ bin and the measurement of $R_{K}$.

For light new physics in the $\mathrm{MeV}$ range a resolution of the $R_{K}$ and $R_{K^{*}}$ measurements in the central $q^{2}$ bin along with other angular observables in $b \rightarrow s \mu^{+} \mu^{-}$decays is possible with the light states coupling only to muons [3, 30, 31]. In addition, in this framework the discrepancy in the anomalous magnetic moment of the muon can also be explained and there are interesting implications for nonstandard neutrino interactions. However, the measurement of $R_{K^{*}}$ in the low $q^{2}$ bin cannot be satisfactorily explained. For the model to work a nontrivial form factor for the flavor changing $b s X$ vertex is required, where $X$ is a light state. This can happen if the $b s X$ coupling is induced at loop level due to some additional light new physics [3] just as we have considered in the case of neutrino scattering. To explain the $R_{K}$ and $R_{K^{*}}$ in all bins with a light mediator is difficult and requires $X$ to couple to electrons rather than muons [3]. In this case the anomalies in the angular observables in $b \rightarrow s \mu^{+} \mu^{-}$decays remain unexplained. This might suggest that there is different new physics responsible for measurements in different $q^{2}$ bins. One can also aim to understand only the low $q^{2}$ bin $R_{K^{*}}$ measurement and such an approach is followed in ref. [32]. It is possible to connect $B$ decays to coherent neutrino scattering by generalizing eq. (2.1) to include all generations of quarks. We write the modified Lagrangian as

$$
\begin{aligned}
\mathcal{L} & =\frac{g}{\Lambda^{2}} \bar{q}_{i}^{\prime} \gamma^{\mu} P_{L, R} Y_{U, D}^{i, j} q_{j}^{\prime} \bar{\chi} \gamma_{\mu}\left(1 \pm \gamma_{5}\right) \chi+i \bar{\chi} \gamma^{\nu}\left[\partial_{\nu}-i g_{\chi} Z^{\prime \nu}\right] \chi-m_{\chi} \bar{\chi} \chi+\frac{1}{2} m_{Z^{\prime}}^{2} Z_{\mu}^{\prime} Z^{\prime \mu} \\
& =H_{\mathrm{eff}}+J_{\mu, \chi} Z^{\prime, \mu}+i \bar{\chi} \gamma^{\nu} \partial_{\nu} \chi-m_{\chi} \bar{\chi} \chi+\frac{1}{2} m_{Z^{\prime}}^{2} Z_{\mu}^{\prime} Z^{\prime \mu}
\end{aligned}
$$

where $i, j$ are the family indices and $Y_{U, D}$ are the flavor couplings for the up and down quarks. To simplify the discussion we assume that only the left-handed quarks are involved in the interactions with the $\chi$ fields. However, in order to satisfy the $R_{K}$ and $R_{K^{*}}$ anomalies we need flavor violation in the $b-s$ sector arising from the following Yukawa matrices:

$$
\begin{aligned}
& Y_{D}=\left(\begin{array}{ccc}
g_{1} & 0 & 0 \\
0 & a_{1} & b_{1} \\
0 & b_{1} & c_{1}
\end{array}\right), \\
& Y_{U}=V_{C K M} Y_{D} V_{C K M}^{\dagger},
\end{aligned}
$$

The origin of the texture in the $Y_{U, D}$ can be understood by introducing a new gauge symmetry motivated by a $\mathrm{U}(1)_{\mu-\tau}$ model [33-36], and including the quark sector. We assume that the Lagrangian has a similar symmetry in the quark sector with the following new Yuakawa terms: $\lambda_{1}^{d} \bar{Q}_{L}^{(2)} \tilde{H}_{3} D_{R}^{(3)}+\lambda_{2} \bar{Q}_{L}^{(3)} \tilde{H}_{4} D_{R}^{(2)}$, where $\tilde{H}_{3,4}$ have new gauge charges 2a, -2 a respectively, in addition to the SM weak charge assignments $(2,1 / 2)$ under $\mathrm{SU}(2)_{L}$ and $\mathrm{U}(1)_{Y}$. Similar terms for the up sector are present as well. Such a model has been constructed in ref. [37]. Here we assume that the quarks transform as $(0, a,-a)$ but we could have assumed $(a, a,-2 a)$ as well with different charge assignments for the new Higgs. 
In the weak interaction basis, the couplings to $Z^{\prime}$ associated with the new symmetry is diagonal,

$$
Y_{U, D}^{\prime}=\left(\begin{array}{ccc}
g_{1} & 0 & 0 \\
0 & g_{2} & 0 \\
0 & 0 & g_{3}
\end{array}\right) .
$$

In transforming from the gauge basis to the mass basis (with the contributions arising from the off-diagonal terms in the Lagrangian), we write

$$
u_{L}^{\prime}=U_{L} u_{L}, \quad d_{L}^{\prime}=D_{L} d_{L},
$$

where $U_{L}$ and $D_{L}$ are $3 \times 3$ unitary matrices for the up and down quarks respectively, and the spinors $u^{(\prime)}$ and $d^{(\prime)}$ include all three generations of fermions. The CKM matrix is given by $V_{C K M}=U_{L}^{\dagger} D_{L}$ and after transforming to the mass basis we can rewrite eq. (5.3) with the $Y_{U, D}^{\prime}$ matrices replaced by $Y_{D}=D_{L}^{\dagger} Y_{D}^{\prime} D_{L}$ and $Y_{U}=U_{L}^{\dagger} Y_{U}^{\prime} U_{L}$. Now if all the mixing is in the up sector with $D_{L}=I$ then there is no FCNC in the down sector. To generate the $b \rightarrow s$ transition we use the assumption of ref. [38] that $D_{L}$ involves only the second and third generations:

$$
D_{L}=\left(\begin{array}{ccc}
1 & 0 & 0 \\
0 & \cos \theta_{D} & \sin \theta_{D} \\
0 & -\sin \theta_{D} & \cos \theta_{D}
\end{array}\right) .
$$

The $Y_{U, D}$ matrices then have the explicit form,

$$
\begin{aligned}
Y_{D} & =\left(\begin{array}{ccc}
g_{1} & 0 & 0 \\
0 & c_{D}^{2} g_{2}+s_{D}^{2} g_{3} & c_{D} s_{D}\left(g_{2}-g_{3}\right) \\
0 & c_{D} s_{D}\left(g_{2}-g_{3}\right) & c_{D}^{2} g_{3}+s_{D}^{2} g_{2}
\end{array}\right), \\
Y_{U} & =V_{C K M} Y_{D} V_{C K M}^{\dagger},
\end{aligned}
$$

where $c_{D} \equiv \cos \theta_{D}$ and $s_{D} \equiv \sin \theta_{D}$. We see that in the down sector flavor changing $b \rightarrow s$ transitions occur with coupling $g_{b s}=c_{D} s_{D}\left(g_{2}-g_{3}\right)$. The form factor for coherent scattering is $F\left(q^{2}\right)=g_{L} \frac{q^{2}}{\Lambda^{2}}$ while for the $B$ decays it is $F\left(q^{2}\right)=g_{L b s} \frac{q^{2}}{\Lambda^{2}}$ with $g_{L} \propto g_{1}, g_{L b s} \propto g_{b s}$ and $\frac{g_{1}}{g_{b s}}=\frac{g_{L}}{g_{L b s}}$. If all the $g_{i}$ are of the same order of magnitude then $g_{L b s}<g_{L}$.

The breakdown of lepton flavor universality required for the $R_{K^{(*)}}$ anomaly can arise in $\mathrm{U}(1)_{\mu-\tau}$ symmetry models. We now compare the flavor violating terms with the flavor conserving terms in the quark sector.

Combining the $B$ decay anomalies with the results from coherent scattering allows us to check for the consistency of this framework. We focus on the $Z^{\prime}$ models. Figure 2 gives the bound on the diagonal term using the COHERENT experiment,

$$
\Lambda^{2}>q_{0}^{2} \frac{g_{L} g_{\mu}}{X_{l}},
$$

where $g_{\mu}$ is the $Z^{\prime}$ coupling to muon neutrinos, and we can read off $X_{l}$ from the figure.

We now turn our attention to the $R_{K^{(*)}}$ anomaly which involves a flavor violating $b-s$ interaction with charged muons. Using the recent results on $R_{K^{(*)}}$, we obtain a constraint 


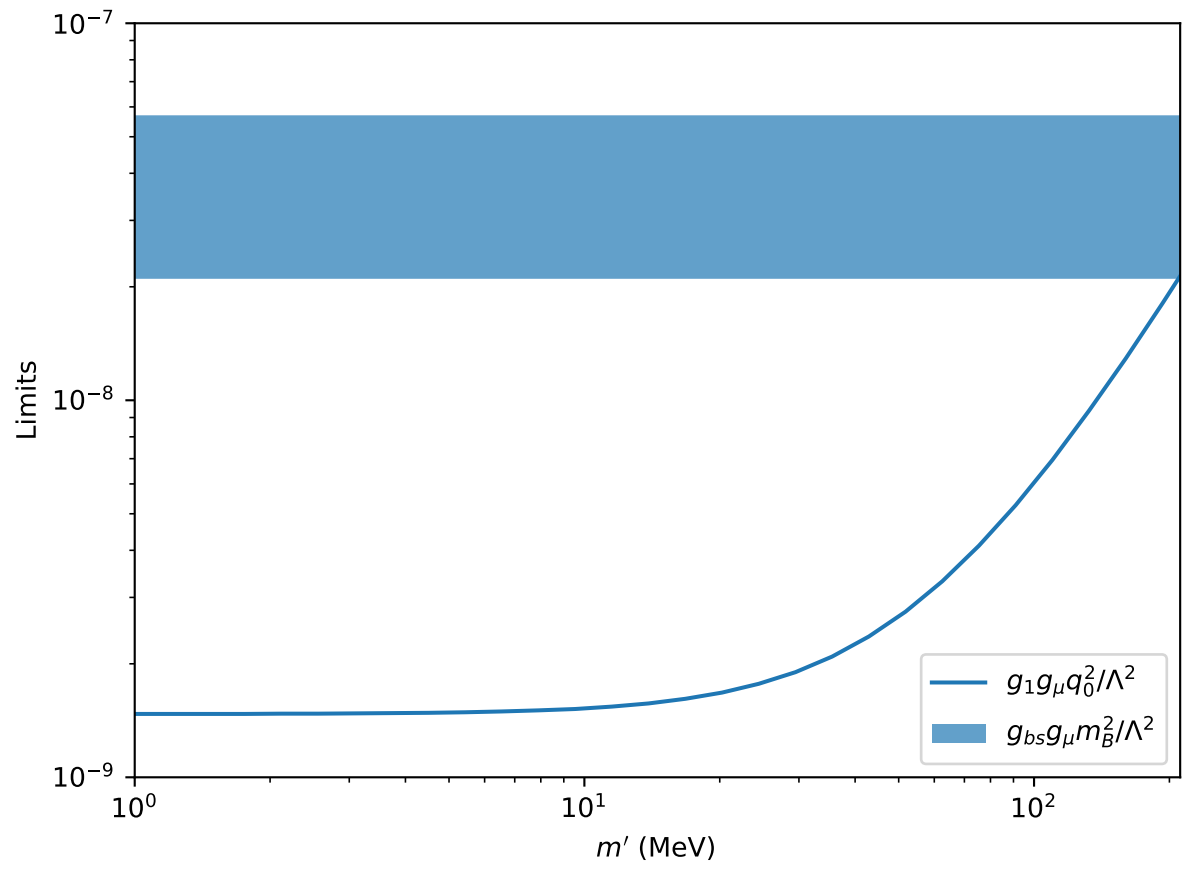

Figure 8. Scale $(\Lambda)$ independent comparison between current $2 \sigma$ COHERENT and $1 \sigma B$ decay constraints on diagonal or off-diagonal couplings for a mediator lighter than $2 m_{\mu}$.

on the flavor violating term. We assume the left handed leptons have identical couplings and so $g_{\mu}$ can be fixed from the muon anomalous magnetic moment measurement and neutrino trident production. Using $g_{\mu} \sim 10^{-3}[3,31]$ and $X_{l} \sim 10^{-9}$ from figure 2 we obtain

$$
\Lambda>10^{3} q_{0} g_{L},
$$

which gives $\Lambda>50 g_{L} \mathrm{GeV}$ for $q_{0}=50 \mathrm{MeV}$. In $B$ decays the relevant $q_{0}$ is taken to be $m_{B}$ and so with the additional assumption of $\mathrm{SU}(2)_{L}$ symmetry for the left handed leptonic couplings we obtain [3],

$$
g_{L b s} \frac{m_{B}^{2}}{\Lambda^{2}} g_{\mu} \sim X_{h}
$$

Combining this with eq. (5.9), we get

$$
\frac{g_{L b s}}{g_{L}}=\frac{g_{b s}}{g_{1}}>\frac{X_{h}}{X_{l}} \frac{q_{0}^{2}}{m_{B}^{2}} .
$$

Using $X_{h} \sim 10^{-8}[3]$ and $X_{l} \sim 10^{-9}$ from figure 8, we find $g_{b s} / g_{1} \sim 10^{-3}$ for $m^{\prime}$ between $1-10 \mathrm{MeV}$, and $g_{b s} / g_{1} \sim 10^{-4}$ for $m^{\prime}=100 \mathrm{MeV}$. However, if the bounds from coherent scattering get stronger, then $\frac{g_{L b s}}{g_{L}}$ will increase and lead to tension in the framework. A similar analysis can also be done with scalar mediators where tighter constraints are obtained. The $B$ anomalies also indicate lepton universality violating new physics which will be interesting to check in neutrino scattering. For instance if the $R_{K^{(*)}}$ anomalies are explained with mediators coupling differently to muons and electrons then $\nu_{\mu}$ and $\nu_{e}$ scattering may show different new physics effects. 


\section{Conclusions}

We have explored the limits of the effective couplings arising from a high energy scale $(\Lambda)$ hidden sector associated with dark matter. We considered two general models which give rise to a coupling form factor that is proportional to the momentum $q^{2}$. The $Z^{\prime}$ model corresponds to vector couplings between neutrinos and quarks, and the $S$ model corresponds to scalar couplings. At low energies, we have shown that it is possible to probe $\Lambda$ via $\mathrm{CE} \nu \mathrm{NS}$ experiments via the form factor which is induced by a DM $(\chi)$ loop. We considered scenarios in which $\Lambda$ is $\geq 50 \mathrm{MeV}$, and in which the energy scale is $\leq 1 \mathrm{MeV}$. CE $\nu \mathrm{NS}$ experiments can probe the former case since $\Lambda$ is higher than the momentum transfer but these experiments are unable to probe the latter case. To probe the scenario with small $\Lambda$, we used solar electron scattering experiments for which the momentum transfer is low.

In the $Z^{\prime}$ model, COHERENT constrains the coupling to be $\sim 10^{-8}$ at $2 \sigma$ for small mediator masses. For large mediator masses, the bound scales according to $\log g^{\prime} \propto 2 \log m^{\prime}$, as shown in figure 2. Atomic parity violation does better than most of the $\mathrm{CE} \nu \mathrm{NS}$ experiments except those using reactor neutrinos. For small $\Lambda$ the Borexino experiment puts $2 \sigma$ constraints on the couplings $\mathcal{O}\left(10^{-7}\right)$ for a $100 \mathrm{MeV}$ mass mediator. Since the momentum transfer is much smaller, the constraints scale like $\log g^{\prime} \propto 2 \log m^{\prime}$ as shown in figure 5 .

In the $S$ model, COHERENT constrains the coupling to be $\sim 10^{-9}$ for small mediator masses. For large mediator masses, the bound scales according to $\log g^{\prime} \propto 2 \log m^{\prime}$, as shown in figure 6. Atomic parity violation experiments do not constrain models with scalar mediators. For small $\Lambda$, the Borexino experiment puts $2 \sigma$ constraints on the couplings $\mathcal{O}\left(10^{-7}\right)$ for a $100 \mathrm{MeV}$ mass mediator. Since the momentum transfer is much smaller, the constraints scale like $\log g^{\prime} \propto 2 \log m^{\prime}$ as shown in figure 6 .

Finally, we have extended our framework to quarks of all generations and have addressed the $R_{K}$ and $R_{K^{*}}$ anomalies in rare $B$ decays. We have shown that a resolution of the anomalies consistent with the present coherent scattering data is possible but future constraints from coherent scattering will provide stringent tests of the $B$ anomalies explanation.

\section{Acknowledgments}

A.D. acknowledges the hospitality of University of California, Irvine where this work was completed. D.M. thanks the Mitchell Institute at Texas A\&M University for its support and hospitality while this work was in progress. A.D. acknowledges support from NSF under Grant No. PHY-1414345. B.D. and L.S. acknowledge support from DOE Grant desc0010813. S.L. acknowledges support from TAMU - College of Science - STRP. D.M. acknowledges support from DOE Grant No. de-sc0010504.

Open Access. This article is distributed under the terms of the Creative Commons Attribution License (CC-BY 4.0), which permits any use, distribution and reproduction in any medium, provided the original author(s) and source are credited. 


\section{References}

[1] COHERENT collaboration, Observation of coherent elastic neutrino-nucleus scattering, Science 357 (2017) 1123 [arXiv: 1708.01294] [INSPIRE].

[2] Borexino collaboration, First simultaneous precision spectroscopy of pp, ${ }^{7}$ Be and pep solar neutrinos with Borexino phase-II, arXiv:1707.09279 [INSPIRE].

[3] A. Datta, J. Kumar, J. Liao and D. Marfatia, New light mediators for the $R_{K}$ and $R_{K^{*}}$ puzzles, Phys. Rev. D 97 (2018) 115038 [arXiv: 1705.08423] [InSPIRE].

[4] J. Goodman, M. Ibe, A. Rajaraman, W. Shepherd, T.M.P. Tait and H.-B. Yu, Constraints on dark matter from colliders, Phys. Rev. D 82 (2010) 116010 [arXiv:1008.1783] [INSPIRE].

[5] Y. Bai, P.J. Fox and R. Harnik, The Tevatron at the frontier of dark matter direct detection, JHEP 12 (2010) 048 [arXiv: 1005.3797] [INSPIRE].

[6] J. Fan, M. Reece and L.-T. Wang, Non-relativistic effective theory of dark matter direct detection, JCAP 11 (2010) 042 [arXiv: 1008.1591] [INSPIRE].

[7] G. Elor, H. Liu, T.R. Slatyer and Y. Soreq, Complementarity for dark sector bound states, Phys. Rev. D 98 (2018) 036015 [arXiv:1801.07723] [InSPIRE].

[8] A. Datta, M. Duraisamy and D. Ghosh, Explaining the $B \rightarrow K^{*} \mu^{+} \mu^{-}$data with scalar interactions, Phys. Rev. D 89 (2014) 071501 [arXiv:1310.1937] [InSPIRE].

[9] D.G. Cerdeño, M. Fairbairn, T. Jubb, P.A.N. Machado, A.C. Vincent and C. Boehm, Physics from solar neutrinos in dark matter direct detection experiments, JHEP 05 (2016) 118 [Erratum ibid. 09 (2016) 048] [arXiv: 1604.01025] [INSPIRE].

[10] M. Cirelli, E. Del Nobile and P. Panci, Tools for model-independent bounds in direct dark matter searches, JCAP 10 (2013) 019 [arXiv:1307.5955] [INSPIRE].

[11] Particle Data Group collaboration, Review of particle physics, Phys. Rev. D 98 (2018) 030001 [INSPIRE].

[12] Y. Nambu and G. Jona-Lasinio, Dynamical model of elementary particles based on an analogy with superconductivity. 1, Phys. Rev. 122 (1961) 345 [INSPIRE].

[13] D. Aristizabal Sierra, B. Dutta and L. Strigari, in preparation (2019).

[14] CONNIE collaboration, The CONNIE experiment, J. Phys. Conf. Ser. 761 (2016) 012057 [arXiv:1608.01565] [INSPIRE].

[15] TEXONO collaboration, Neutrino and dark matter physics with sub-KeV Germanium detectors, J. Phys. Conf. Ser. 718 (2016) 062036 [INSPIRE].

[16] MINER collaboration, Background studies for the MINER coherent neutrino scattering reactor experiment, Nucl. Instrum. Meth. A 853 (2017) 53 [arXiv:1609.02066] [InSPIRE].

[17] COHERENT collaboration, COHERENT 2018 at the spallation neutron source, arXiv: 1803.09183 [INSPIRE].

[18] DAYA BAY collaboration, Improved measurement of the reactor antineutrino flux and spectrum at Daya Bay, Chin. Phys. C 41 (2017) 013002 [arXiv:1607.05378] [InSPIRE].

[19] Super-Kamiokande collaboration, Solar neutrino results in Super-Kamiokande-III, Phys. Rev. D 83 (2011) 052010 [arXiv:1010.0118] [INSPIRE]. 
[20] SNO collaboration, Combined analysis of all three phases of solar neutrino data from the Sudbury Neutrino Observatory, Phys. Rev. C 88 (2013) 025501 [arXiv:1109.0763] [INSPIRE].

[21] W.C. Haxton, R.G. Hamish Robertson and A.M. Serenelli, Solar neutrinos: status and prospects, Ann. Rev. Astron. Astrophys. 51 (2013) 21 [arXiv:1208.5723] [InSPIRE].

[22] B. Bhattacharya, A. Datta, D. London and S. Shivashankara, Simultaneous explanation of the $R_{K}$ and $R\left(D^{(*)}\right)$ puzzles, Phys. Lett. B 742 (2015) 370 [arXiv:1412.7164] [InSPIRE].

[23] A. Greljo, G. Isidori and D. Marzocca, On the breaking of lepton flavor universality in $B$ decays, JHEP 07 (2015) 142 [arXiv: 1506.01705] [INSPIRE].

[24] A. Crivellin, D. Müller and T. Ota, Simultaneous explanation of $R\left(D^{(*)}\right)$ and $b \rightarrow s \mu^{+} \mu^{-}$: the last scalar leptoquarks standing, JHEP 09 (2017) 040 [arXiv:1703.09226] [INSPIRE].

[25] LHCb collaboration, Test of lepton universality with $B^{0} \rightarrow K^{* 0} \ell^{+} \ell^{-}$decays, JHEP 08 (2017) 055 [arXiv:1705.05802] [INSPIRE].

[26] G. Hiller and F. Krüger, More model-independent analysis of $b \rightarrow s$ processes, Phys. Rev. D 69 (2004) 074020 [hep-ph/0310219] [INSPIRE].

[27] G. Hiller and M. Schmaltz, $R_{K}$ and future $b \rightarrow$ sll physics beyond the Standard Model opportunities, Phys. Rev. D 90 (2014) 054014 [arXiv: 1408.1627] [InSPIRE].

[28] LHCb collaboration, Test of lepton universality using $B^{+} \rightarrow K^{+} \ell^{+} \ell^{-}$decays, Phys. Rev. Lett. 113 (2014) 151601 [arXiv:1406.6482] [INSPIRE].

[29] M. Bordone, G. Isidori and A. Pattori, On the Standard Model predictions for $R_{K}$ and $R_{K^{*}}$, Eur. Phys. J. C 76 (2016) 440 [arXiv:1605.07633] [INSPIRE].

[30] A. Datta, J. Liao and D. Marfatia, A light $Z^{\prime}$ for the $R_{K}$ puzzle and nonstandard neutrino interactions, Phys. Lett. B 768 (2017) 265 [arXiv:1702.01099] [INSPIRE].

[31] A.K. Alok, B. Bhattacharya, A. Datta, D. Kumar, J. Kumar and D. London, New physics in $b \rightarrow s \mu^{+} \mu^{-}$after the measurement of $R_{K^{*}}$, Phys. Rev. D 96 (2017) 095009 [arXiv: 1704.07397] [INSPIRE].

[32] W. Altmannshofer et al., Light resonances and the low- $q^{2}$ bin of $R_{K^{*}}$, JHEP 03 (2018) 188 [arXiv: 1711.07494] [INSPIRE].

[33] C.S. Lam, A 2-3 symmetry in neutrino oscillations, Phys. Lett. B 507 (2001) 214 [hep-ph/0104116] [INSPIRE].

[34] T. Kitabayashi and M. Yasue, $S_{2 L}$ permutation symmetry for left-handed $\mu$ and $\tau$ families and neutrino oscillations in an $\mathrm{SU}(3)_{L} \times \mathrm{SU}(1)_{N}$ gauge model, Phys. Rev. D 67 (2003) 015006 [hep-ph/0209294] [INSPIRE].

[35] W. Grimus and L. Lavoura, A discrete symmetry group for maximal atmospheric neutrino mixing, Phys. Lett. B 572 (2003) 189 [hep-ph/0305046] [INSPIRE].

[36] Y. Koide, Universal texture of quark and lepton mass matrices with an extended flavor $2 \leftrightarrow 3$ symmetry, Phys. Rev. D 69 (2004) 093001 [hep-ph/0312207] [InSPIRE].

[37] A. Datta and P.J. O'Donnell, The 2-3 symmetry: flavor changing $b, \tau$ decays and neutrino mixing, Phys. Rev. D 72 (2005) 113002 [hep-ph/0508314] [INSPIRE].

[38] B. Bhattacharya, A. Datta, J.-P. Guévin, D. London and R. Watanabe, Simultaneous explanation of the $R_{K}$ and $R_{D^{(*)}}$ puzzles: a model analysis, JHEP 01 (2017) 015 [arXiv: 1609.09078] [INSPIRE]. 